\title{
I nvestigation of carbonaceous interstellar dust analogues by infrared spectroscopy: Effects of energetic processing
}

\author{
Belén Maté, \\ Miguel J iménez-Redondo, Isabel Tanarro, Miguel Moreno, and \\ Victor Herrero \\ Instituto de Estructura de la Materia (IEM-CSIC), Serrano 123, 28006, \\ Madrid, Spain \\ belen.mate@csic.es
}

ISMS - 70TH MEETING - JUNE 22-26, 2015 - CHAMPAI GN-URBANA, ILLINOIS

Funding: CDS2009-00038, FIS20012 -38175, FIS2013-48087-C2-1-P, ERC-2013-Syg 610256 


\section{I nterstellar hydrocarbon dust}

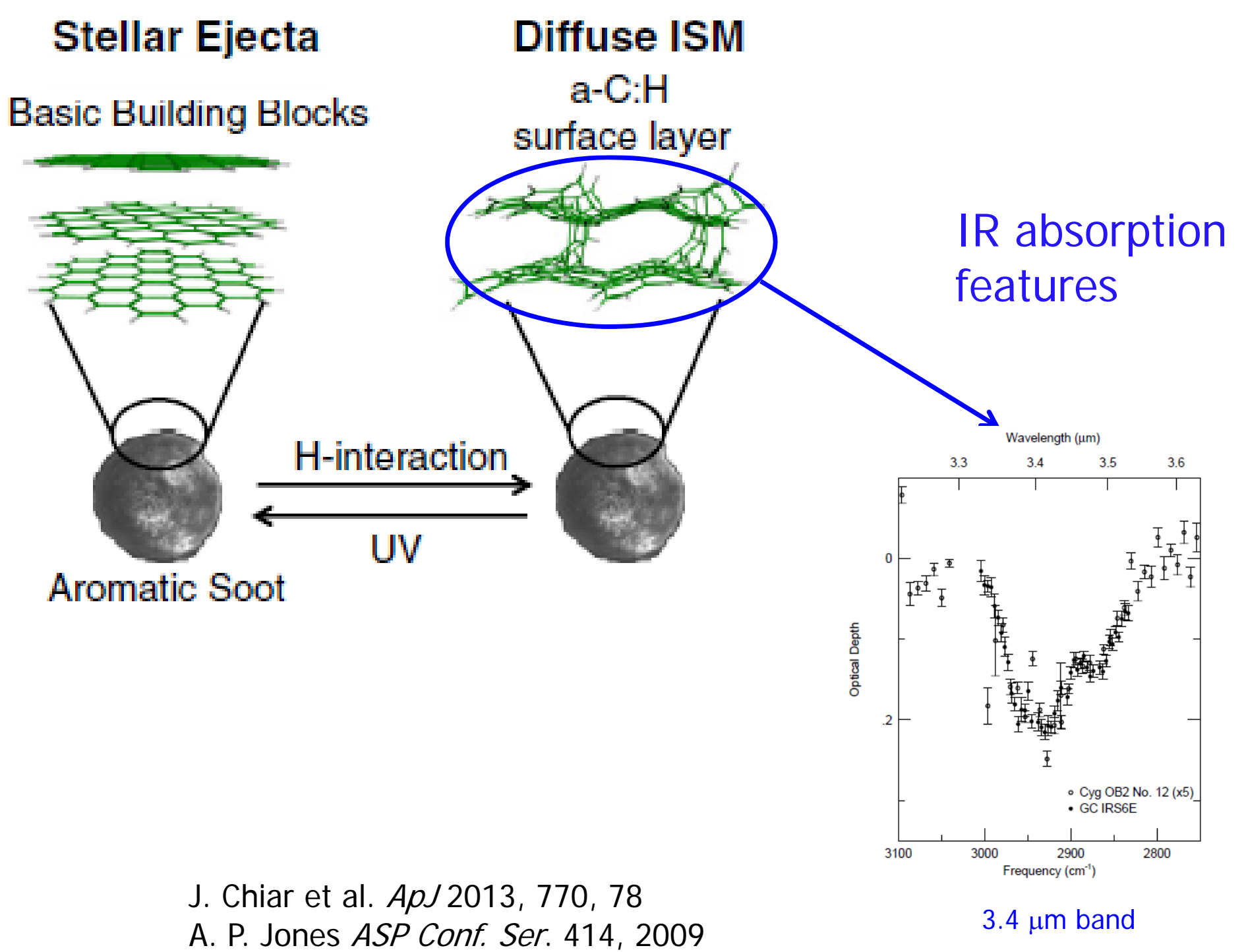




\section{Carbonaceous dust in the diffuse I SM}
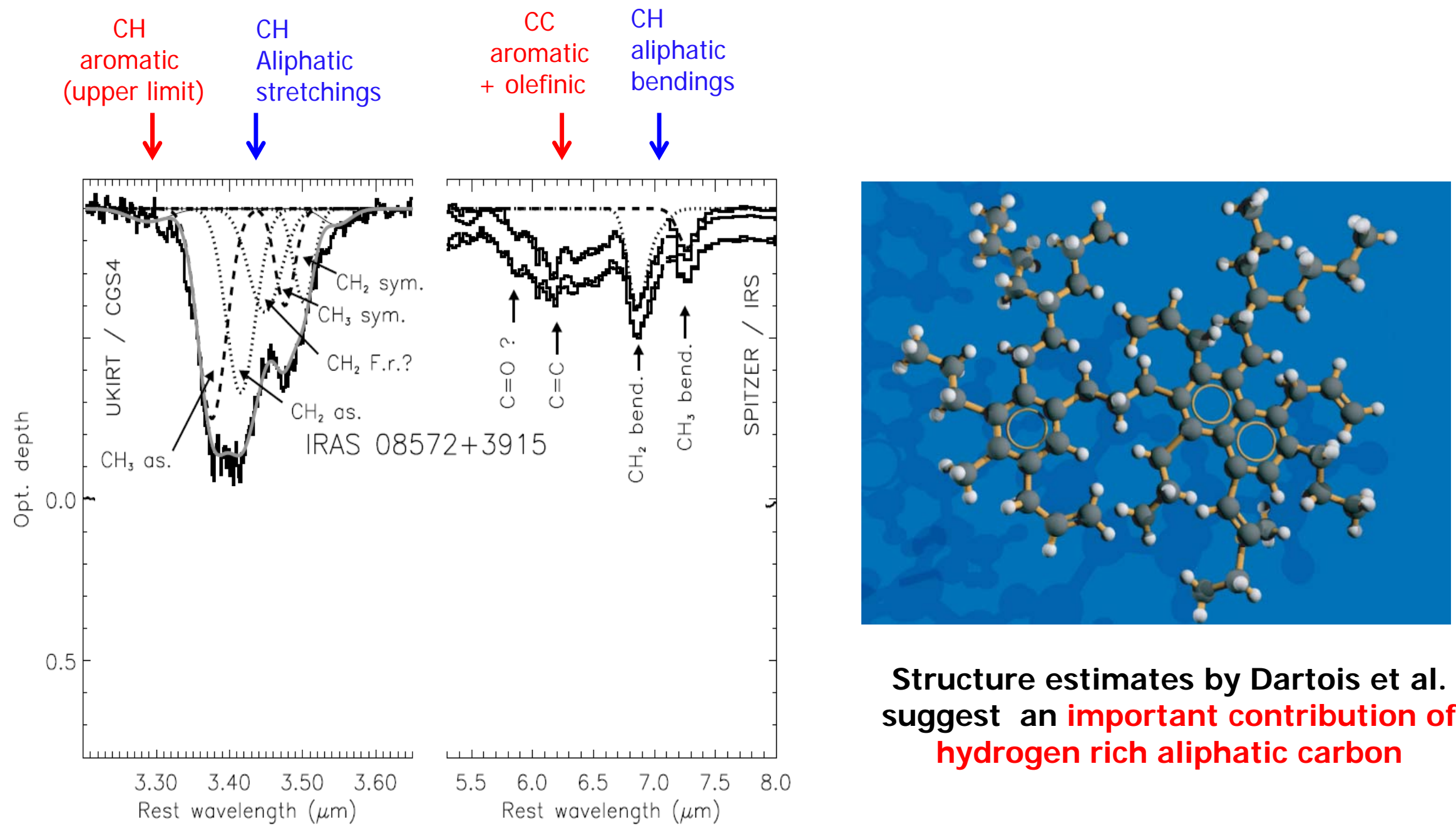

Structure estimates by Dartois et al. suggest an important contribution of hydrogen rich aliphatic carbon

Aliphatic features are more prominent

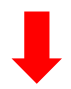

their absorption strengths are much higher

E. Dartois et al. $A \& A, 2007,463,635$ 


\section{Amorphous $\mathrm{C} / \mathrm{H}$ ternary diagram}

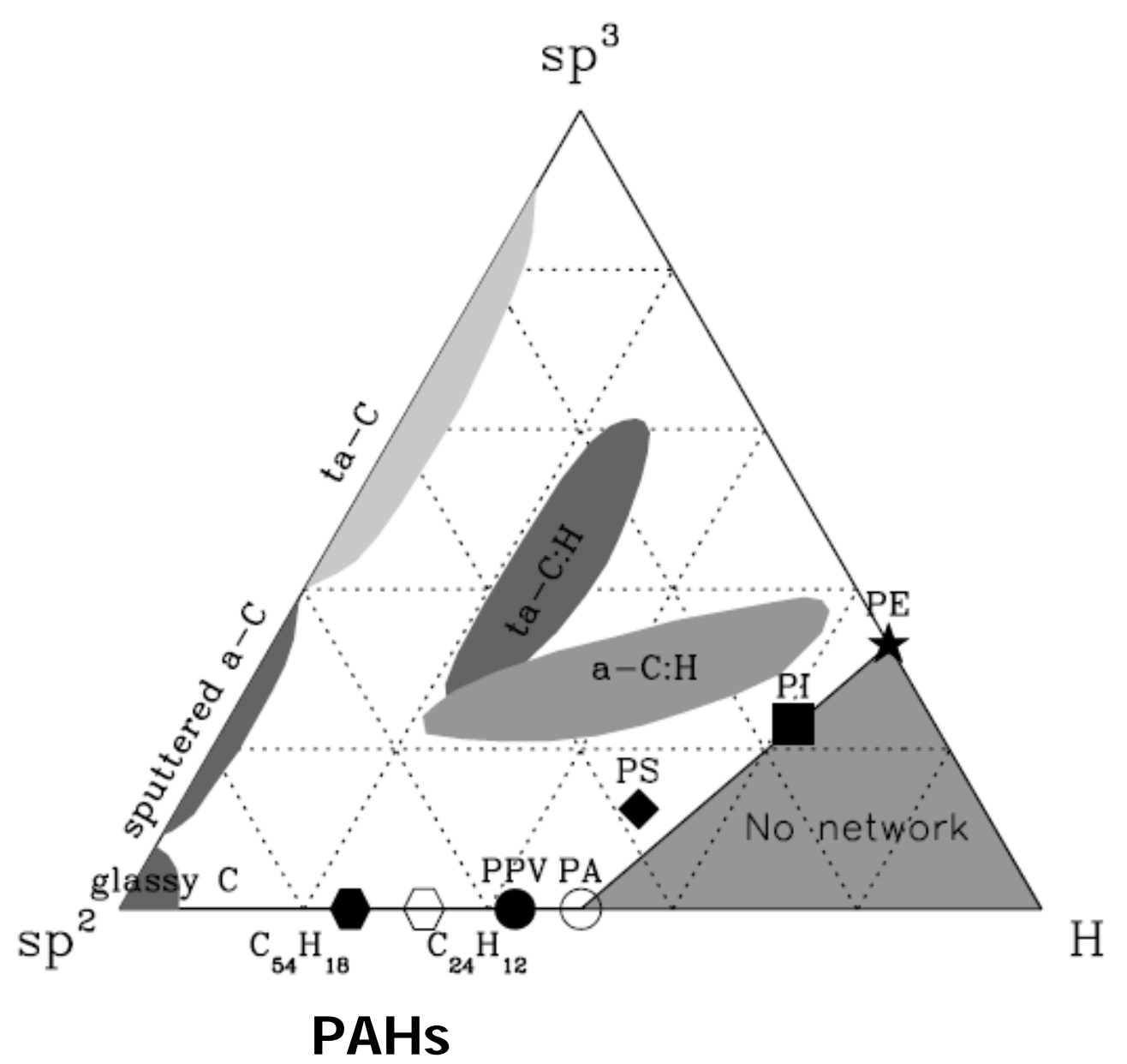

E. Dartois et al. $A \& A, 2007,463,635$

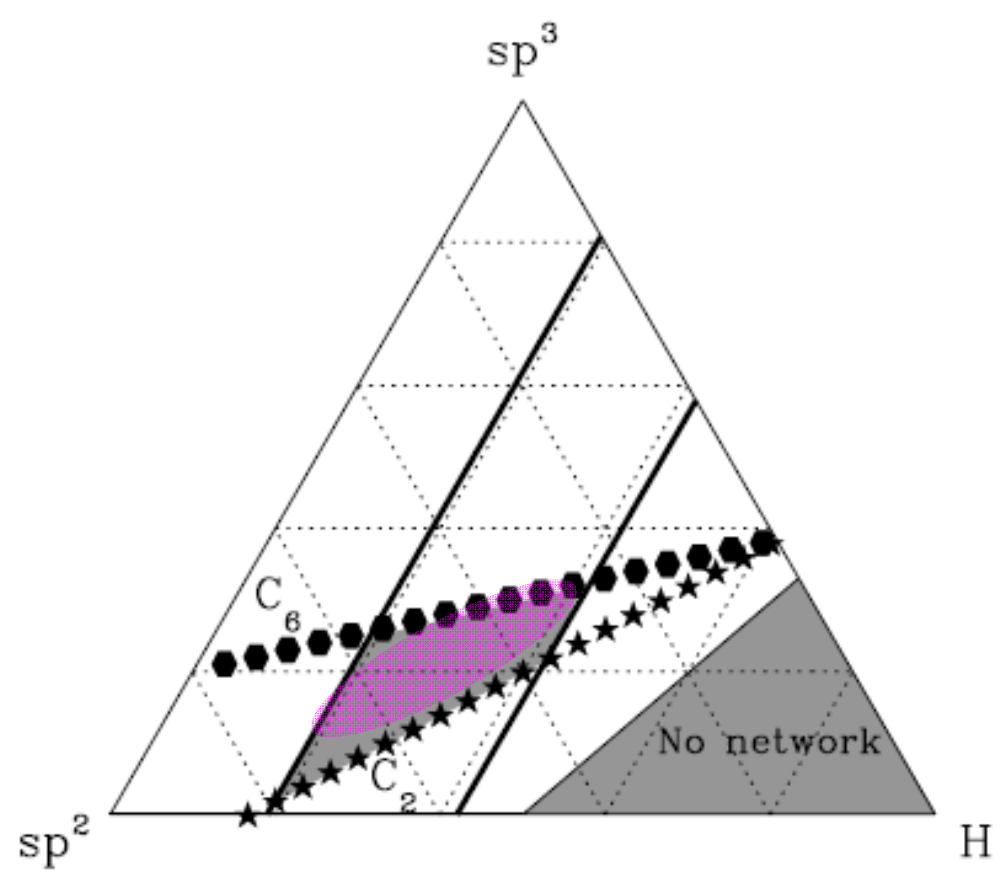




\section{Carbonaceous dust in the diffuse ISM}
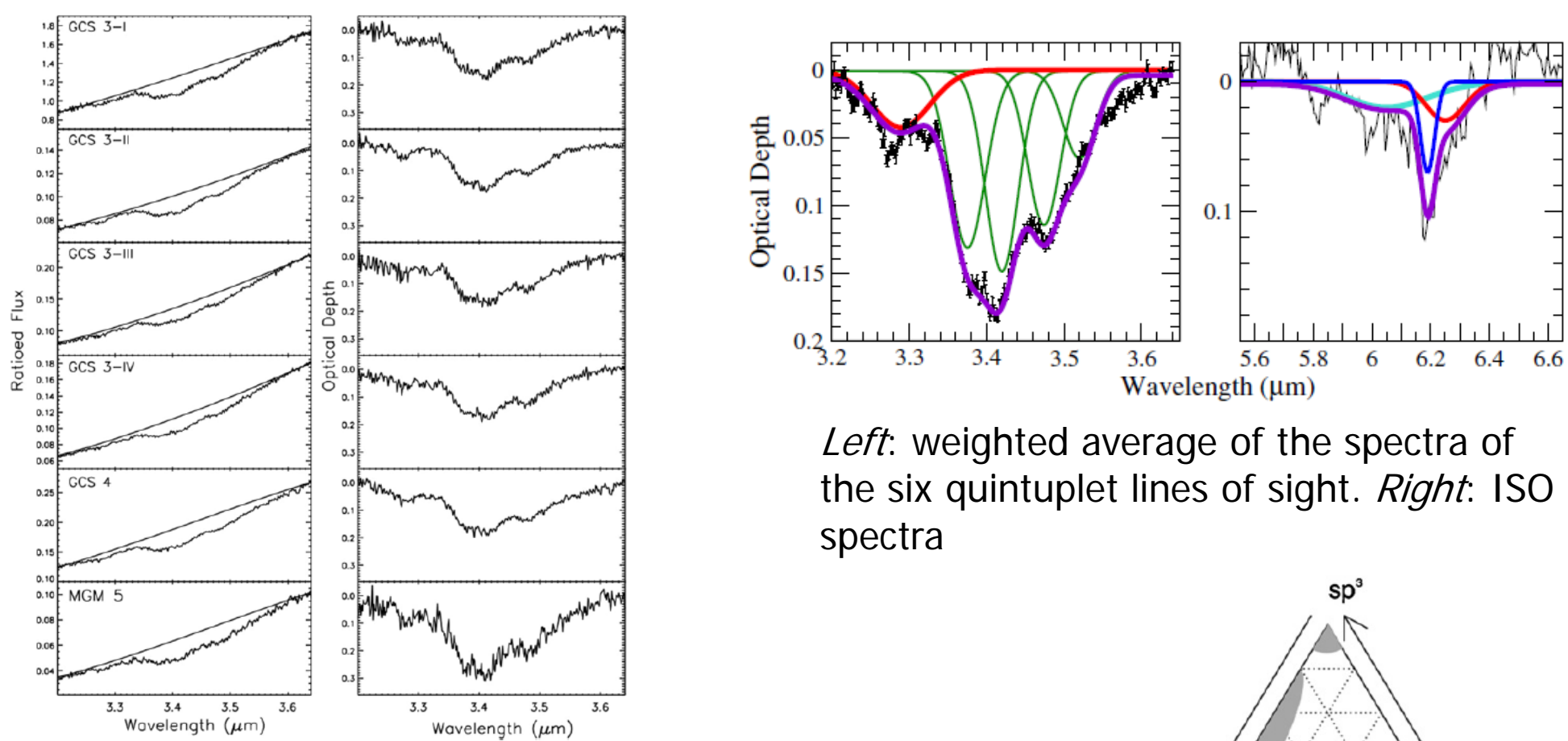

Left: weighted average of the spectra of the six quintuplet lines of sight. Right. ISO spectra

UKIRT spectra toward the Galactic center Quintuplet Cluster

Structure estimates by Chiar et al. suggest that the dust is hydrogen poor and highly aromatic

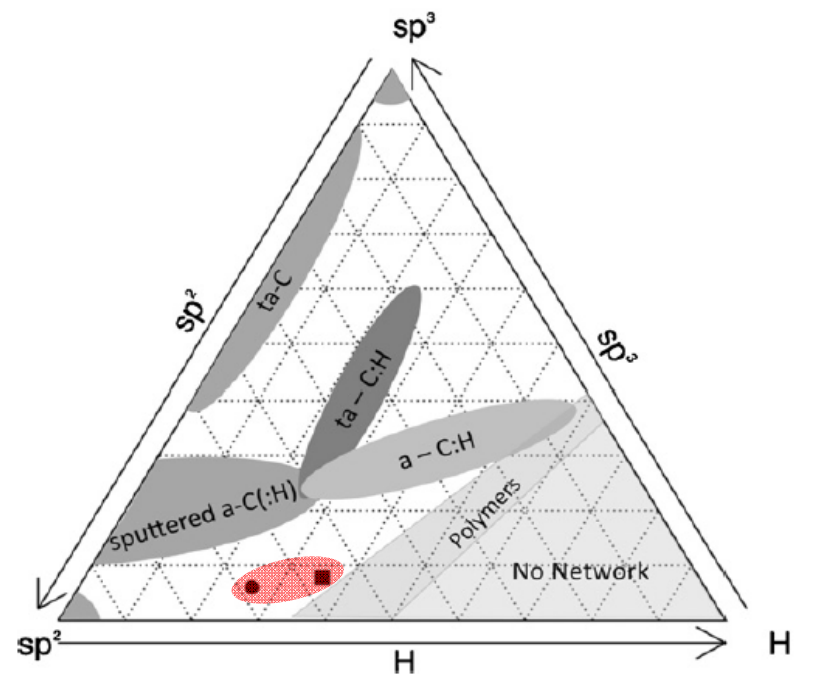

J. Chiar et al. $A p J, 2013,770,78$ 


\section{HAC film deposition in $\mathrm{CH}_{4} /$ He plasma IEM-CSIC}

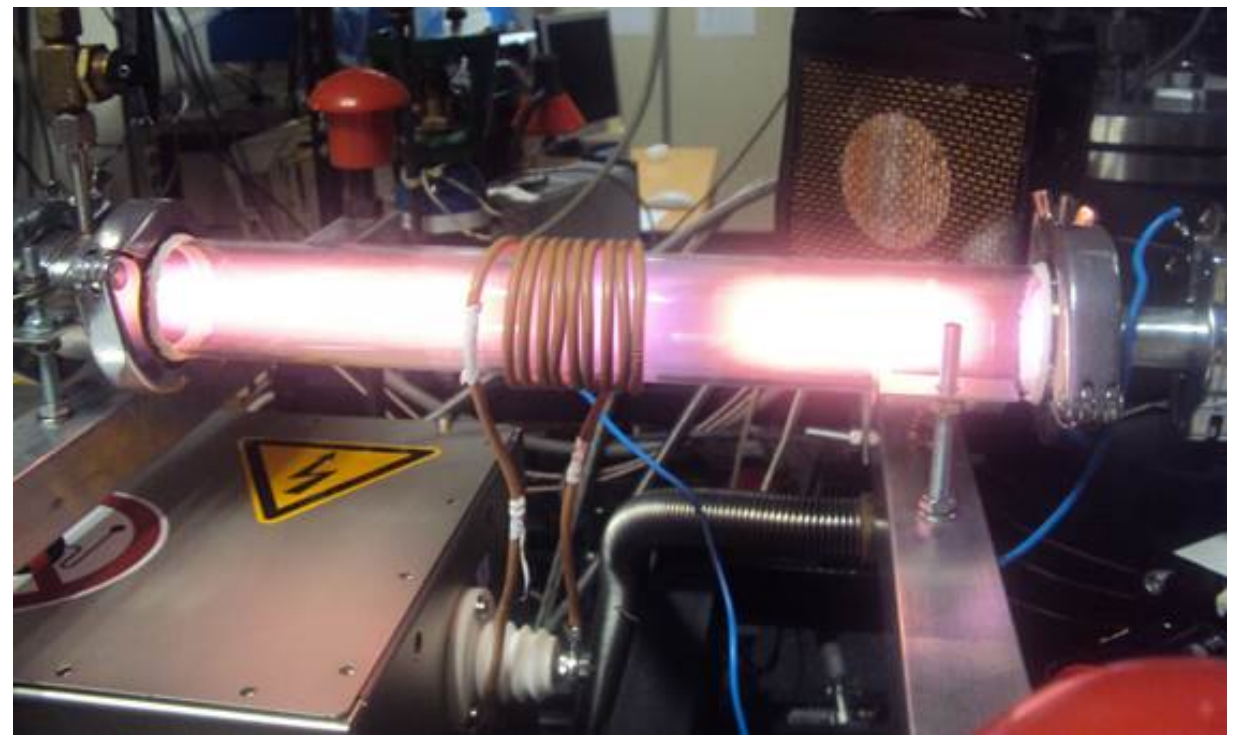

Inductively coupled RF discharge reactor

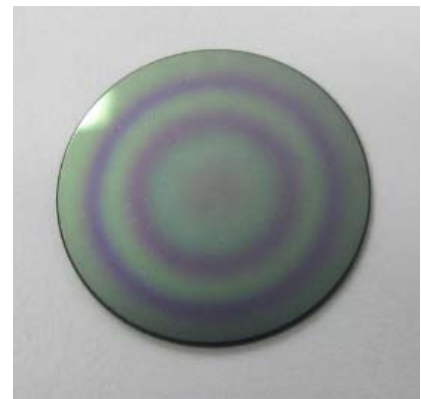

outside the coil

HAC1

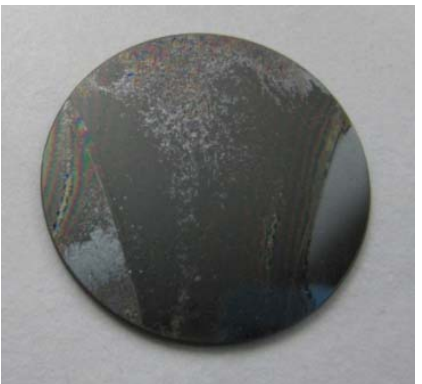

inside the coil

HAC2
- $\mathrm{CH}_{4}(40 \%) / \mathrm{He}$ 0.3 mbar

- $40 \mathrm{~W}, 13.56 \mathrm{MHz}$
Film thickness $\sim 3 \mu \mathrm{m}$ 1 hour 


\section{R spectra of the deposits}

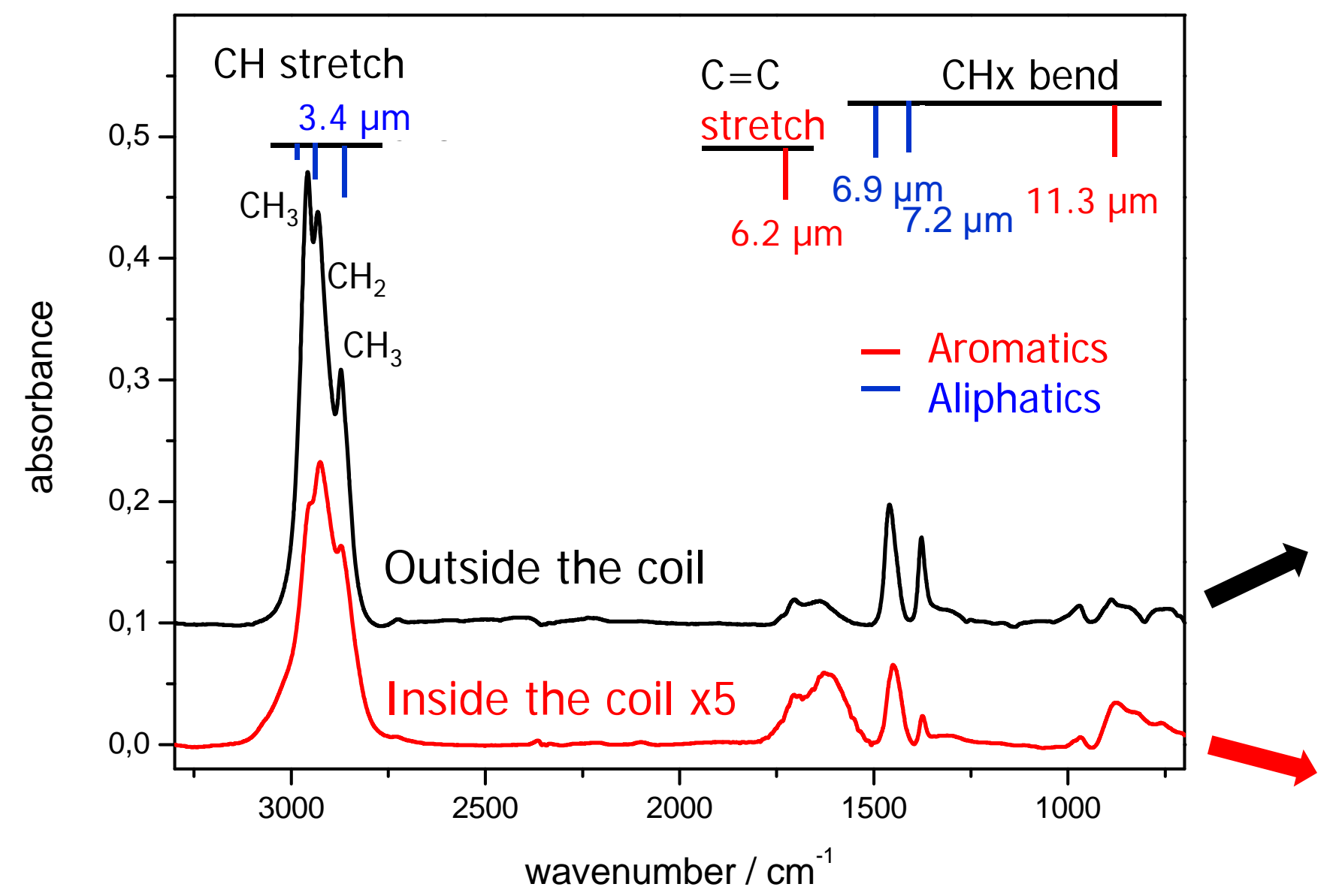

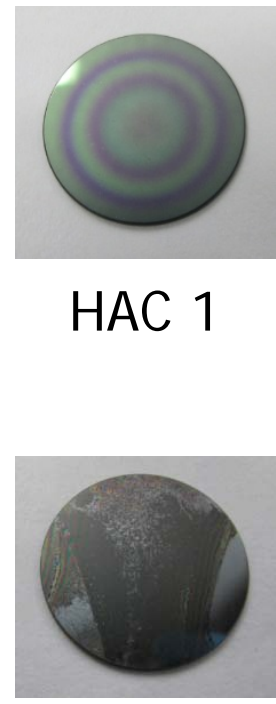

HAC 2

B. Maté et al. Faraday Disc. 2014, 168, 267 


\section{Analysis of HAC spectra}

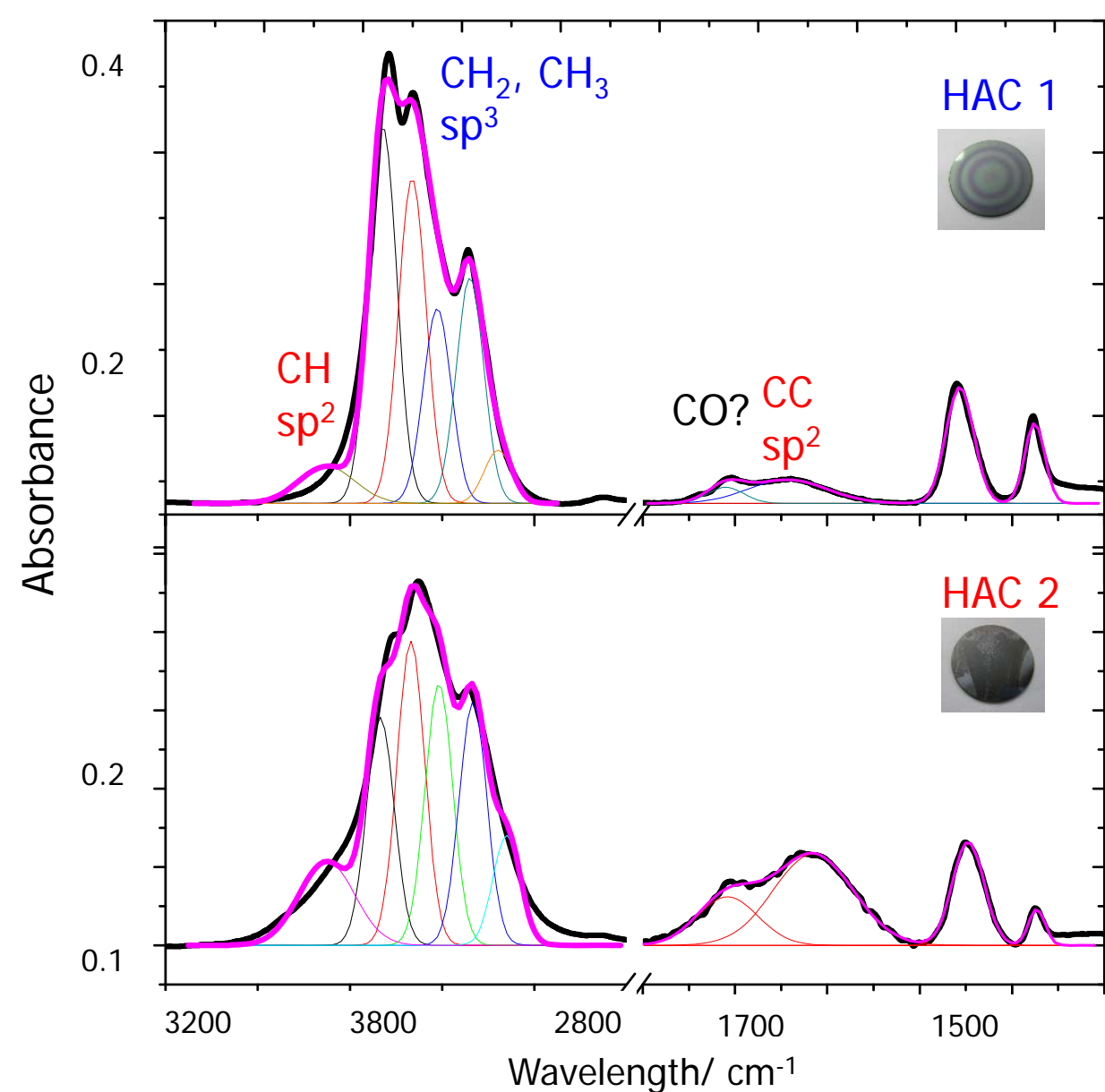

Using absorption strengths from Chiar et al. $A p J$ 2013, 770, 78

\begin{tabular}{|l|l|}
\hline $\mathrm{H}$ & 0.33 \\
\hline $\mathrm{sp}^{2}$ & 0.55 \\
\hline $\mathrm{sp}^{3}$ & 0.11 \\
\hline
\end{tabular}

\begin{tabular}{|l|l|}
\hline $\mathrm{H}$ & 0.13 \\
\hline $\mathrm{sp}^{2}$ & 0.83 \\
\hline $\mathrm{sp}^{3}$ & 0.04 \\
\hline
\end{tabular}

1300

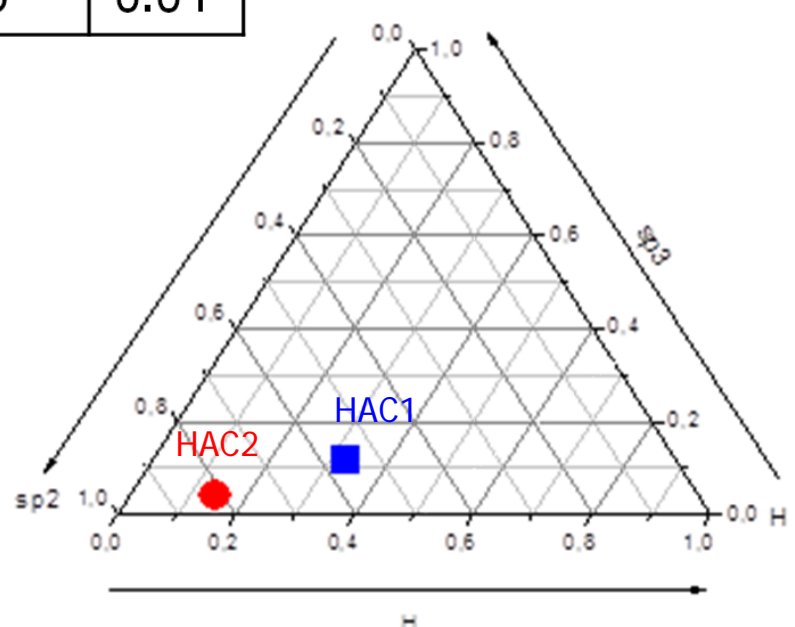




\section{Comparison with observations in the diffuse ISM}

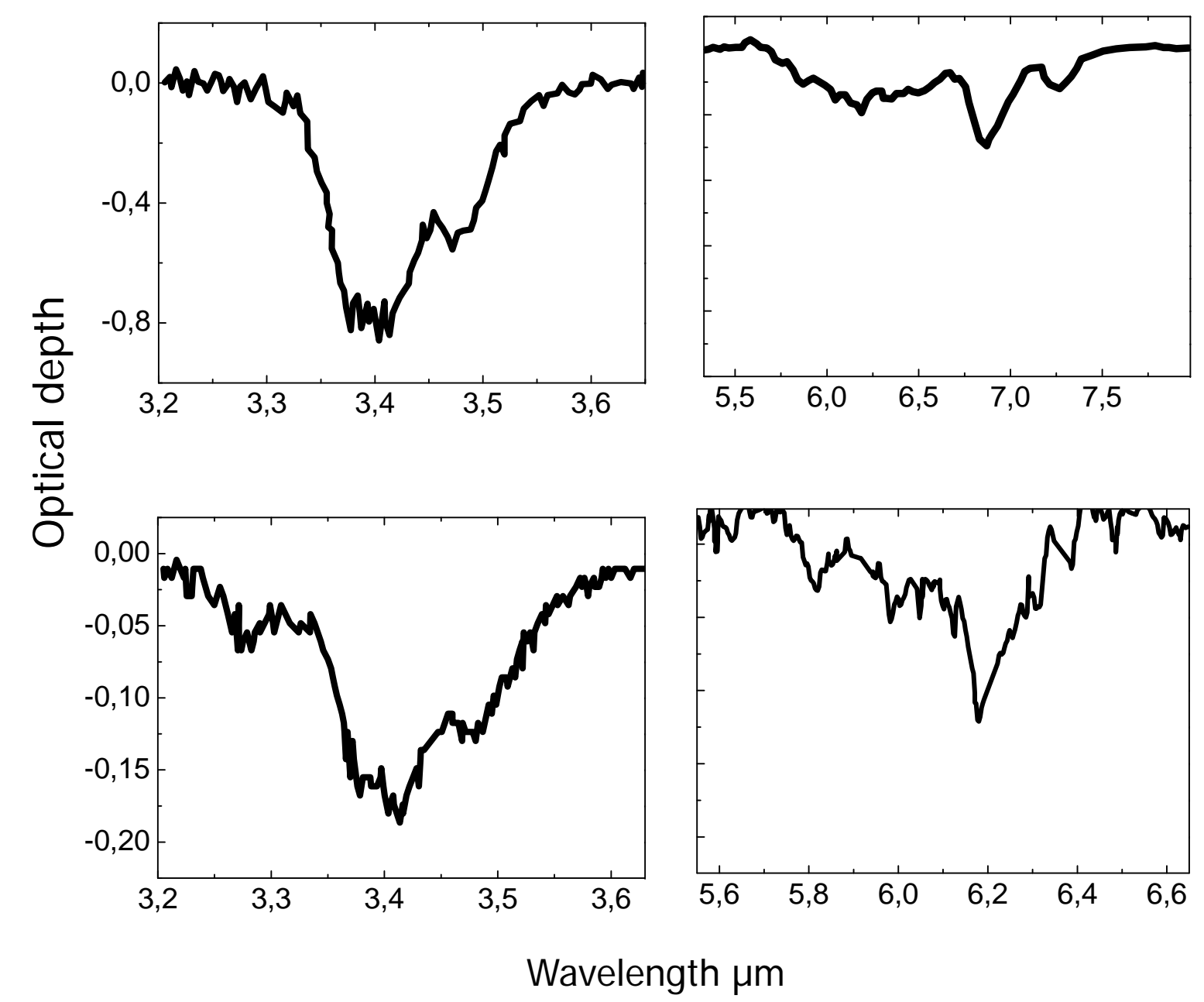

Infrared Galaxy IRAS

$08572+3915$

Dartois et al. A\&A 2007

Galactic center Quintuplet Cluster.

Chiar et al. ApJ , 2013, 


\section{Comparison with observations in the diffuse ISM}
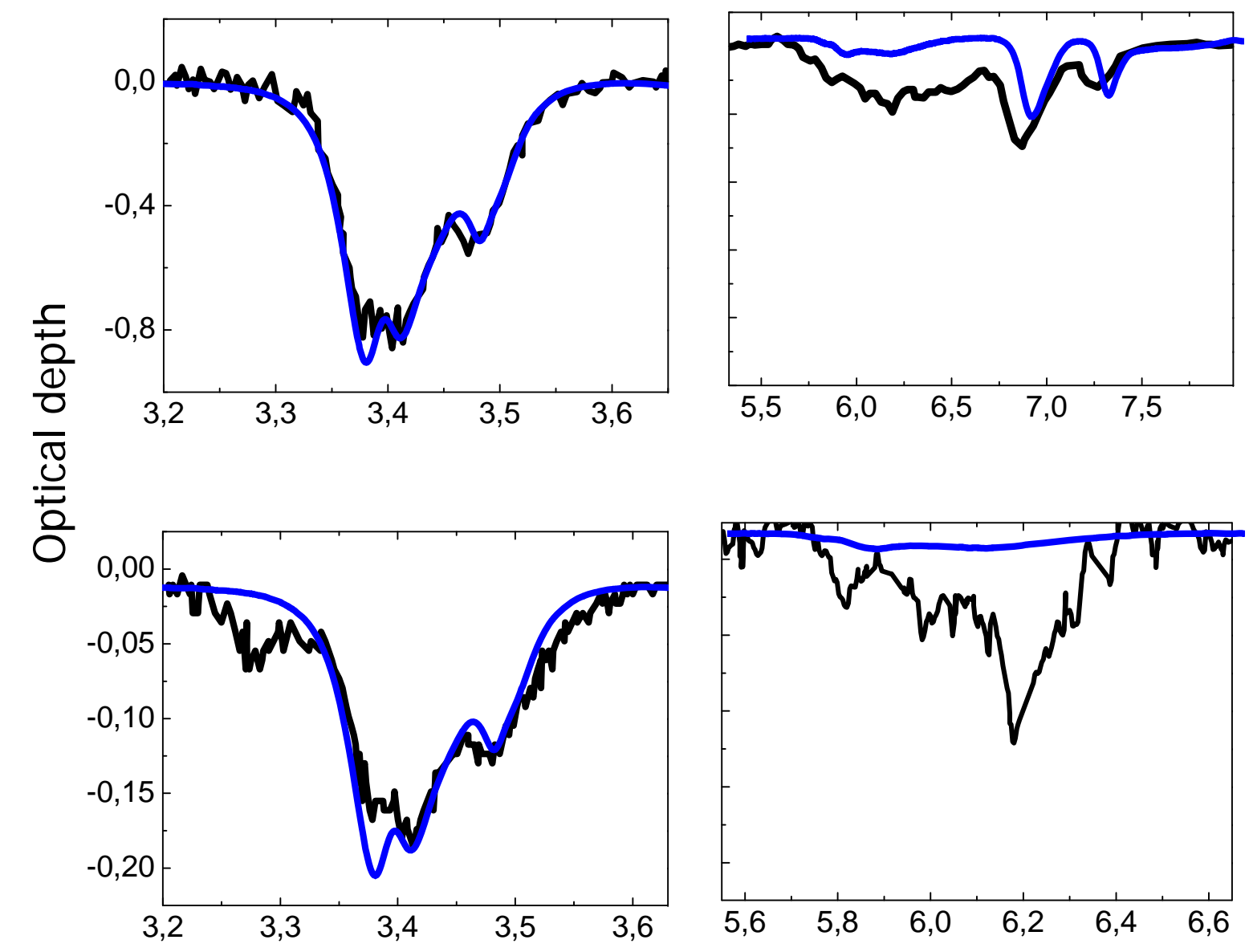

I nfrared Galaxy IRAS

$08572+3915$

Dartois et al. A\&A 2007

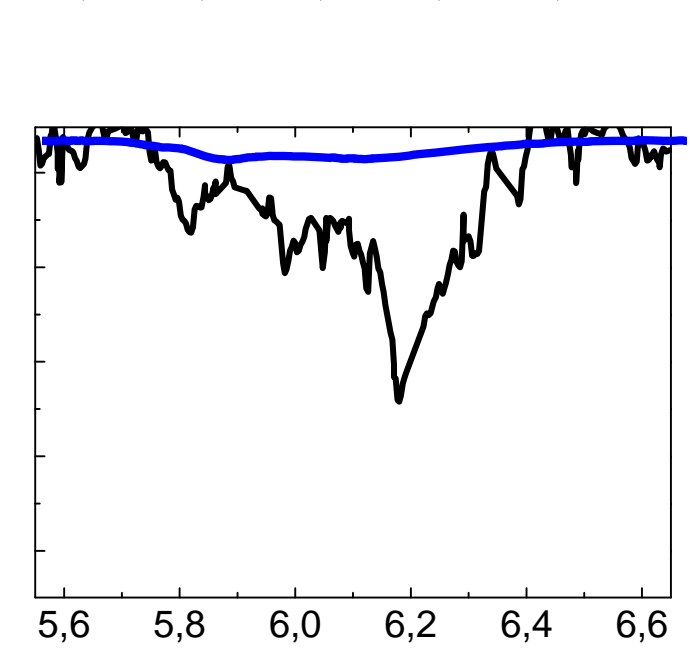

HAC 1 spectrum scaled to the maximum

Galactic center Quintuplet Cluster.

Chiar et al. ApJ , 2013,

Wavelength $\mu \mathrm{m}$ 


\section{Comparison with observations in the diffuse ISM}
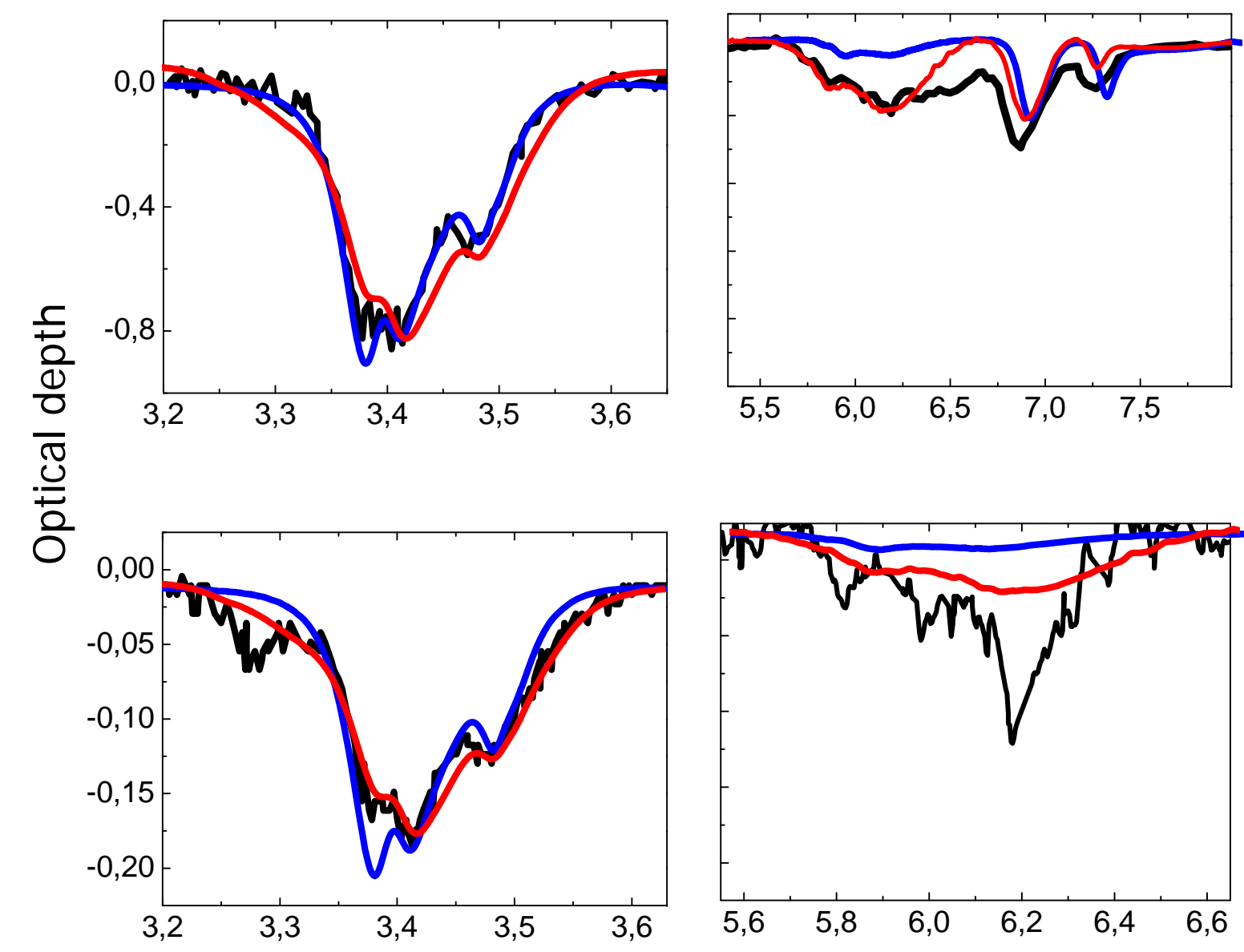

Infrared Galaxy IRAS

08572+3915

Dartois et al. A\&A 2007

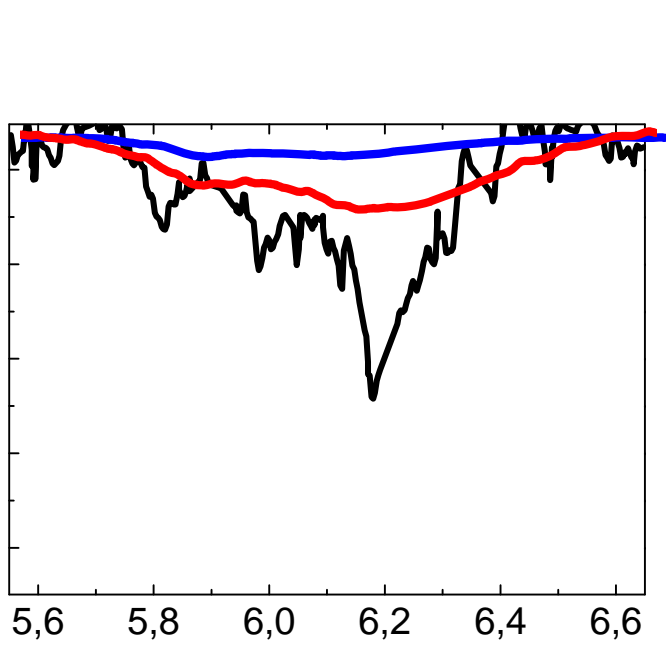

HAC 1 spectrum scaled to the maximum

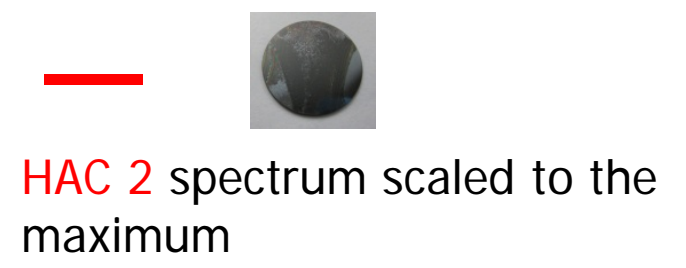

Galactic center Quintuplet Cluster.

Chiar et al. ApJ , 2013,

Wavelength $\mu \mathrm{m}$ 


\section{Deposit growth and structure}
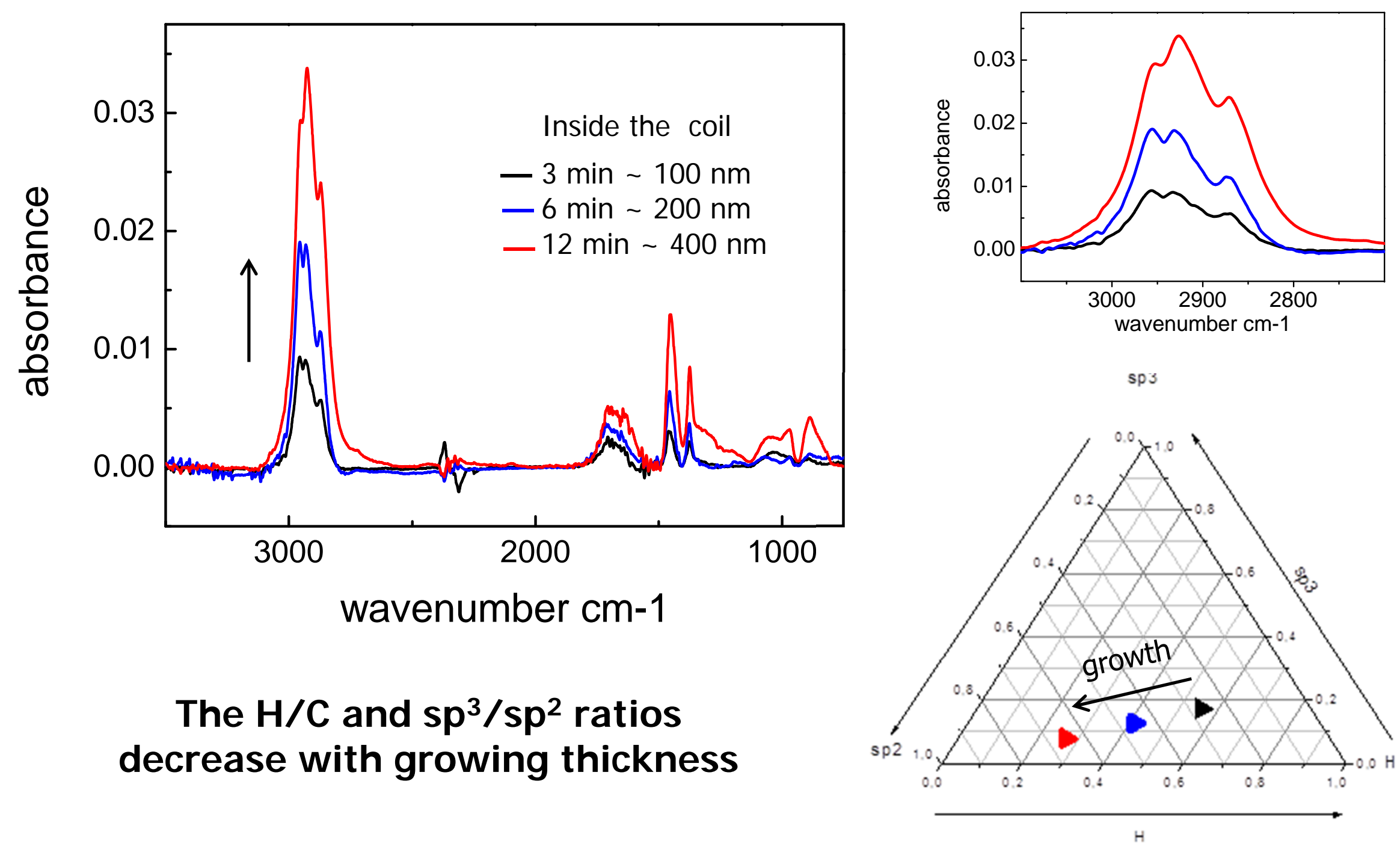

The $\mathrm{H} / \mathrm{C}$ and $\mathrm{sp}^{3} / \mathrm{sp}^{2}$ ratios decrease with growing thickness 


\section{Processing in $\mathbf{H}_{2}$ plasma}

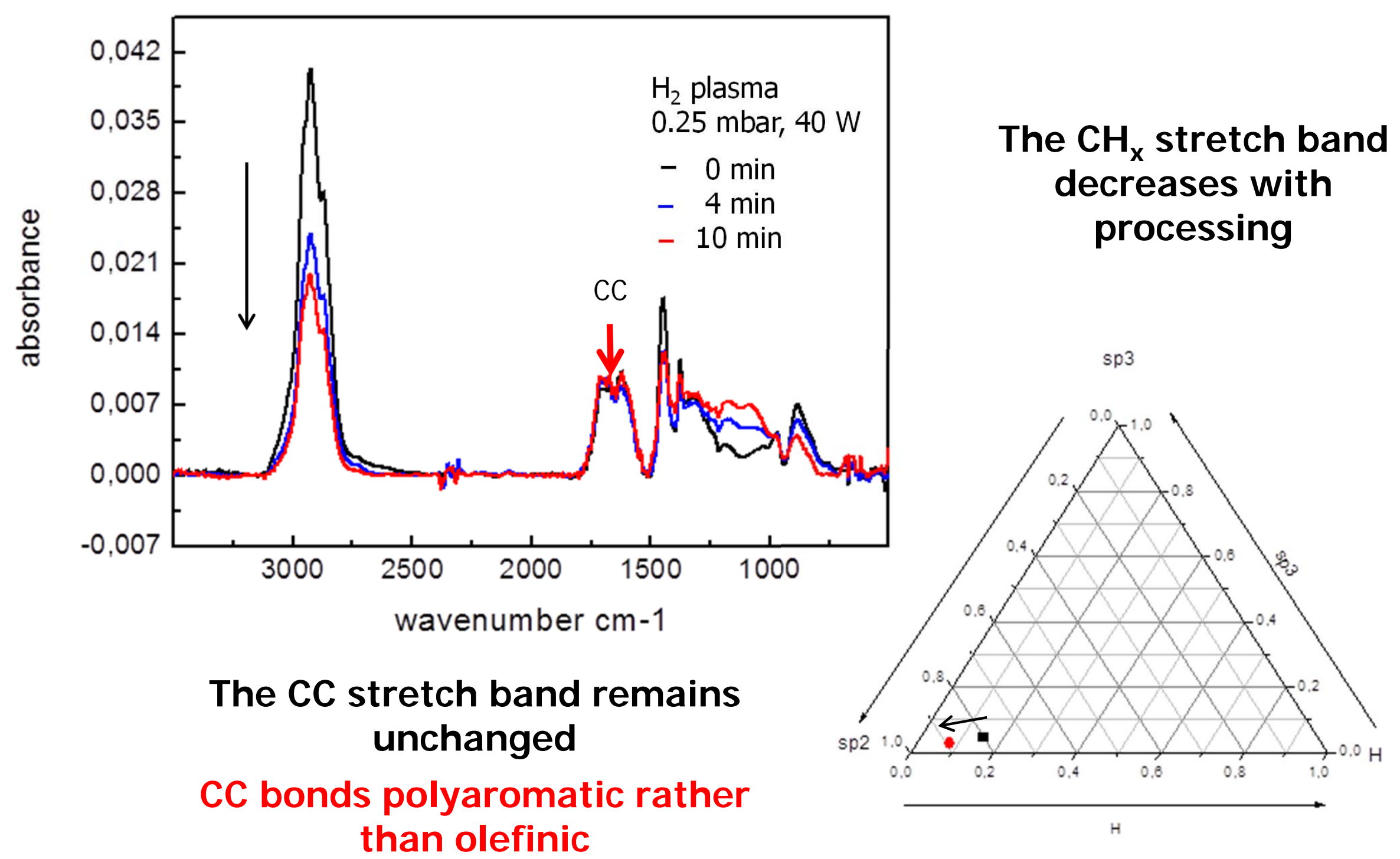




\section{Summary and conclusions}

- Carbonaceous dust analogues have been grown in inductively coupled RF discharges of $\mathrm{He}+\mathrm{CH}_{4}$.

- The position in the plasma reactor affects the structure of the deposited hydrocarbon. Inside coil deposits are good analogues to interstellar medium dust.

- Processing with $\mathrm{H}_{2}$ plasma do not affect the aromatic component of the carbonaceous material. 